\title{
On inverse eigenvalue problems for block Toeplitz matrices with Toeplitz blocks*
}

\author{
Zhongyun $\operatorname{Liu}^{\dagger}$ \\ School of Mathematics and Computing Science, Changsha University of Science \\ and Technology, Changsha, Hunan, 410076, P. R. China \\ Yulin Zhang, C. Ferreira and Rui Ralha \\ Department of Mathematics, University of Minho, Campus de Gualtar, 4710-057 \\ Braga, Portugal.
}

\begin{abstract}
We propose an algorithm for solving the inverse eigenvalue problem for real symmetric block Toeplitz matrices with symmetric Toeplitz blocks. It is based upon an algorithm which has been used before by others to solve the inverse eigenvalue problem for general real symmetric matrices and also for Toeplitz matrices. First we expose the structure of the eigenvectors of the so-called generalized centrosymmetric matrices. Then we explore the properties of the eigenvectors to derive an efficient algorithm that is able to deliver a matrix with the required structure and spectrum. We have implemented our ideas in a Matlab code. Numerical results produced with this code are included.
\end{abstract}

Keywords: Block Toeplitz matrix, generalized $K$-centrosymmetric matrix, inverse eigenvalue problem, Newton method

AMS Classifications: 15A18, 15A42, 65F15

\section{Introduction}

Inverse eigenvalue problem (IEP) concerns the reconstruction of a matrix from prescribed spectral data. Due to their remarkable variety of applications ranging from applied mechanics and physics to numerical analysis, inverse eigenvalue problems have intrigued the researchers for decades. See $[4,6,8]$. In this paper we will study the inverse eigenvalue problem for real symmetric block Toeplitz (RSBT) matrices.

Let $T \in \mathbb{R}^{n \times n}$ be a real $l \times l$ block Toeplitz matrix,

$$
T=\left[\begin{array}{ccccc}
T_{0} & T_{1} & \cdots & \cdots & T_{l-1} \\
T_{-1} & T_{0} & T_{1} & \cdots & \cdots \\
\cdots & \ddots & \ddots & \ddots & \cdots \\
\cdots & \cdots & T_{-1} & T_{0} & T_{1} \\
T_{-l+1} & \cdots & \cdots & T_{-1} & T_{0}
\end{array}\right]
$$

where $\left\{T_{i}\right\}_{i=-l+1}^{l-1} \in \mathbb{R}^{k \times k}$ and $k l=n$. We say that $T$ is symmetric block Toeplitz with symmetric Toeplitz blocks if $T$ is block symmetric, that is, $T_{i}=T_{-i}$, and if each block $T_{i}, i=0,1, \cdots, l-1$, is symmetric and Toeplitz.

\footnotetext{
${ }^{*}$ Supported by the National Natural Science Foundation of China, No. 10771022 and 10571012 , Scientific Research Foundation for the Returned Overseas Chinese Scholars, State Education Ministry of China, No. 890 [2008], and Major Foundation of Educational Committee of Hunan Province, No. 09A002 [2009], the Portuguese Foundation for Science and Technology (FCT) through the Programme POCI 2010, respectively.

${ }^{\dagger}$ Corresponding author. E-mail:liuzhongyun@263.net
} 
For example, when $n=6$ and $l=2$, the size of the blocks is $k=3$ and, for $\alpha_{i}, \beta_{i} \in \mathbb{R}$,

$$
T=\left[\begin{array}{ccc|ccc}
\alpha_{1} & \alpha_{2} & \alpha_{3} & \beta_{1} & \beta_{2} & \beta_{3} \\
\alpha_{2} & \alpha_{1} & \alpha_{2} & \beta_{2} & \beta_{1} & \beta_{2} \\
\alpha_{3} & \alpha_{2} & \alpha_{1} & \beta_{3} & \beta_{2} & \beta_{1} \\
\hline \beta_{1} & \beta_{2} & \beta_{3} & \alpha_{1} & \alpha_{2} & \alpha_{3} \\
\beta_{2} & \beta_{1} & \beta_{2} & \alpha_{2} & \alpha_{1} & \alpha_{2} \\
\beta_{3} & \beta_{2} & \beta_{1} & \alpha_{3} & \alpha_{2} & \alpha_{1}
\end{array}\right] .
$$

Such class of matrices arises naturally in many applications, such as signal processing, trigonometric moment problems, queueing problems, integral equations and elliptic partial differential equations with boundary conditions solved by means of finite differences. See, for example, [3, 14, 16, 17, 24].

A RSBT matrix $T$ with symmetric Toeplitz blocks can be parameterized as

$$
T(\mathbf{c})=\sum_{i=1}^{n} c_{i} S_{i}
$$

where $\mathbf{c}=\left[\begin{array}{llll}c_{1} & c_{2} & \ldots & c_{n}\end{array}\right]^{T} \in \mathbb{R}^{n}$ and $S_{i}$ are certain $n \times n$ real symmetric matrices.

We denote the eigenvalues of $T(\mathbf{c})$ by

$$
\lambda_{i}(\mathbf{c}), i=1, \ldots, n, \quad \text { with } \quad \lambda_{1}(\mathbf{c}) \leq \cdots \leq \lambda_{n}(\mathbf{c}) .
$$

Then the inverse eigenvalue problem under consideration can be described as

Problem 1 Given real numbers $\lambda_{1}^{*} \leq \lambda_{2}^{*} \leq \ldots \leq \lambda_{n}^{*}$ and $l \in \mathbb{N}$, find $\mathbf{c} \in \mathbb{R}^{n}$ such $T(\mathbf{c})$ is an $l \times l$ RSBT matrix with symmetric Toeplitz blocks and $\lambda_{i}(\mathbf{c})=\lambda_{i}^{*}$, for $i=1, \ldots, n$.

We call $\lambda_{i}^{*}, i=1, \ldots, n$, the target eigenvalues and $\Lambda^{*}=\left\{\lambda_{1}^{*}, \ldots, \lambda_{n}^{*}\right\}$ the target spectrum.

Problem 1 is sometimes called a parameterized inverse eigenvalue problem (PIEP). See $[6,12]$. There exists a large amount of literature concerning the conditions for the existence and uniqueness of the solutions to the PIEP (or its variations) in many special cases. See, for example, $[2,4,6,8]$ and references therein.

Regarding the PIEP as a nonlinear system of $n$ equations in $n$ unknowns, a natural strategy would be a Newton-type iteration. Several numerical methods based on Newton iteration for PIEP were presented in [12], assuming the existence of a solution. These methods have been studied and developed for structured IEP's like the case of real symmetric Toeplitz matrices. See $[5,8,25]$.

When $k=1$ or $l=1$, the RSBT matrix $T$ in (1.1) reduces to a general real symmetric Toeplitz (RST) matrix. In this case, Problem 1 becomes the standard IEP for RST matrices, which has been paid much attention for years. See, for instance, $[5,7,9,10,11,13,19,20,21,25]$. Therefore, Problem 1 for RSBT matrices is a generalization of the IEP for RST matrices.

The numerical methods for a PIEP presented in [12] can be adapted to solve Problem 1. However, those schemes do not explore the structure of block Toeplitz matrices and their eigenvector properties. In order to solve Problem 1 more efficiently, it is necessary to design a structure-preserving algorithm. In this paper we will present an algorithm that respects this property.

The remainder of this paper is organized as follows: in the next section we recall some facts about generalized $K$-centrosymmetric matrices and discuss the reduced form and the eigenvector structure of T. In section 3 we describe a structurepreserving algorithm that is based on results presented in $[5,6,8]$. Some numerical examples are shown in Section 4. 


\section{Preliminaries}

Throughout this paper, we denote by $I_{n}$ the identity matrix of order $n$; $J_{n}$ the antidiagonal or reversal matrix (ones on the cross diagonal, bottom left to top right, and zeros elsewhere); $K$ a fixed permutation matrix of order $n$ consisting of the product of disjoint transpositions; $\Pi_{l, k}$ the $l \times l$ block anti-diagonal matrix with $I_{k}$ on the cross block diagonal and zeros elsewhere and $\Theta_{l, k}$ the $l \times l$ block diagonal matrix with $J_{k}$ on the block diagonal and zeros elsewhere. Thus, we have

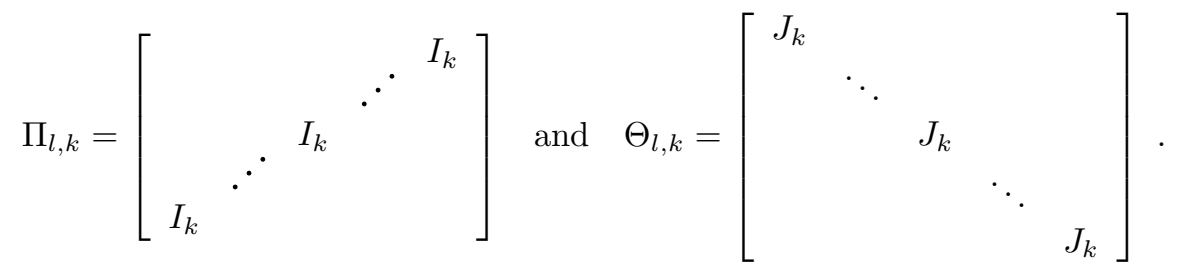

\subsection{Reduction of generalized centrosymmetric matrices}

We first discuss the reduced structure of generalized centrosymmetric matrices.

Definition $1[1,18,22,23] A$ matrix $A \in \mathbb{R}^{n \times n}$ is said to be centrosymmetric if $A=J_{n} A J_{n}$ and generalized $K$-centrosymmetric if $A=K A K$.

Some useful facts about $n \times n$ generalized $K$-centrosymmetric matrices can be found in [22]. In particular, it is shown that every $n \times n$ generalized $K$ centrosymmetric matrix can be reduced to a $2 \times 2$ block diagonal matrix by a simple similarity transformation.

Lemma 1 [22, Theorem 1] A matrix $A \in \mathbb{R}^{n \times n}$ is a generalized $K$-centrosymmetric matrix if and only if there exists an orthogonal matrix $Q$ such that

$$
Q^{T} A Q=\operatorname{diag}(M, N),
$$

where $M \in \mathbb{R}^{(n-l) \times(n-l)}$ and $N \in \mathbb{R}^{l \times l}$ with $l=\operatorname{rank}(I-K)$.

Definition 2 Let $A \in \mathbb{R}^{n \times n}$ be an $l \times l$ block matrix with each block of order $k$. A is said to be an $l \times l$ block centrosymmetric matrix if $A=\Pi_{l, k} A \Pi_{l, k}$ and an $l \times l$ block-wise centrosymmetric matrix if $A=\Theta_{l, k} A \Theta_{l, k}$.

Block centrosymmetric matrices and block-wise centrosymmetric matrices are two special cases of generalized $K$-centrosymmetric matrices [22].

A RSBT matrix $T$ with symmetric Toeplitz blocks is simultaneously centrosymmetric, block centrosymmetric and block-wise centrosymmetric. We can make use of all these properties to reduce $T$ into the direct sum of 4 matrices.

For convenience, we denote by $\mathcal{B}_{l}$ the set of $l \times l$ block centrosymmetric matrices; $\mathcal{W}_{l}$ the set of $l \times l$ block-wise centrosymmetric matrices; $\mathcal{G}_{l}$ the intersection $\mathcal{B}_{l} \cap \mathcal{W}_{l}$ and $\mathcal{C}_{n}$ the set of $n \times n$ centrosymmetric matrices.

It can be shown that if $A \in \mathcal{B}_{l}$, then $A$ can be partitioned into the following form:

$$
A=\left\{\begin{array}{l|l}
{\left[\begin{array}{c|c}
B & \Pi_{s, k} C \Pi_{s, k} \\
\hline C & \Pi_{s, k} B \Pi_{s, k}
\end{array}\right],} & l=2 s \\
{\left[\begin{array}{c|c|c}
B & \Pi_{s, k} E & \Pi_{s, k} C \Pi_{s, k} \\
\hline F & G & F \Pi_{s, k} \\
\hline C & E & \Pi_{s, k} B \Pi_{s, k}
\end{array}\right],} & l=2 s+1
\end{array},\right.
$$

where $B, C \in \mathbb{R}^{s k \times s k}, G \in \mathbb{R}^{k \times k}, E \in \mathbb{R}^{s k \times k}$ and $F \in \mathbb{R}^{k \times s k}$. 
According to [22], the orthogonal matrix $Q$ in Lemma 1 is given by

$$
Q=\frac{\sqrt{2}}{2}\left\{\begin{array}{c|c}
{\left[\begin{array}{c|c}
I_{s k} & I_{s k} \\
\hline \Pi_{s, k} & -\Pi_{s, k}
\end{array}\right],} & l=2 s \\
{\left[\begin{array}{c|c|c}
I_{s k} & & I_{s k} \\
\hline & \sqrt{2} I_{k} & \\
\hline \Pi_{s, k} & & -\Pi_{s, k}
\end{array}\right],} & l=2 s+1
\end{array}\right.
$$

Thus, using Lemma 1 we can straightforward derive the following result:

Corollary 1 Let matrix $Q$ be given by (2.3). Then $A \in \mathcal{B}_{l}$ if and only if $Q^{T} A Q=$ $\operatorname{diag}(M, N)$, where

$$
M=\left\{\begin{array}{cc}
B+\Pi_{s, k} C, & l=2 s \\
{\left[\begin{array}{cc}
B+\Pi_{s, k} C & \sqrt{2} \Pi_{s, k} E \\
\sqrt{2} F & G
\end{array}\right],} & l=2 s+1
\end{array}\right.
$$

and

$$
N=B-\Pi_{s, k} C
$$

Similarly, if $A \in \mathcal{W}_{l}$, define

$$
\hat{Q}=\frac{\sqrt{2}}{2}\left[\begin{array}{c|c|c|c|c|c|c|c}
I_{t} & & & & I_{t} & & & \\
J_{t} & & & & -J_{t} & & & \\
\hline & I_{t} & & & & I_{t} & & \\
& J_{t} & & & & -J_{t} & & \\
\hline & & \ddots & & & & \ddots & \\
\hline & & & I_{t} & & & & I_{t} \\
& & & J_{t} & & & & -J_{t}
\end{array}\right], \quad \text { if } k=2 t,
$$

and

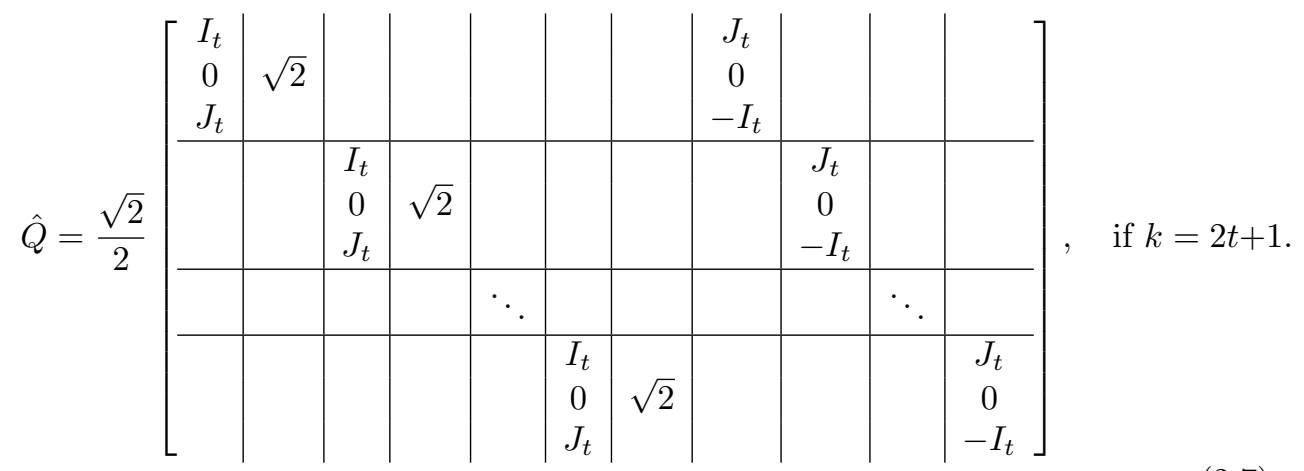

Now we have the following result which is an immediate conclusion of Lemma 1 with $K=\Theta_{l, k}$. For more details, see [22].

Corollary 2 Let matrix $\hat{Q}$ be defined by (2.6) or (2.7). Then $A \in \mathcal{W}_{l}$ if and only if $\hat{Q}^{T} A \hat{Q}=\operatorname{diag}(M, N)$.

Block centrosymmetric matrices, block-wise centrosymmetric matrices and centrosymmetric matrices are related as the next lemma describes. 
Lemma 2 Assume that $A \in \mathbb{R}^{n \times n}$ is an $l \times l$ block matrix with each block of order $k$. Then any two of the three statements

$$
\text { (a) } A \in \mathcal{B}_{l}, \quad(b) A \in \mathcal{W}_{l} \quad \text { and } \quad(c) A \in \mathcal{C}_{n}
$$

imply that the third one holds.

Proof. Assume that (a) and (b) hold. Thus, $A \in \mathcal{G}_{l}$, that is, $A=\Pi_{l, k} A \Pi_{l, k}$ and $A=\Theta_{l, k} A \Theta_{l, k}$. Because $\Pi_{l, k} \Theta_{l, k}=\Theta_{l, k} \Pi_{l, k}=J_{n}$, we conclude that $A$ is centrosymmetric. For the other two cases, we only need to use the fact that $\Theta_{l, k} J_{n}=$ $J_{n} \Theta_{l, k}=\Pi_{l, k}$ and $\Pi_{l, k} J_{n}=J_{n} \Pi_{l, k}=\Theta_{l, k}$.

For $A \in \mathcal{G}_{l}$, define

$$
P=Q \operatorname{diag}\left(Q_{1}, Q_{2}\right)
$$

where $Q$ is given in (2.3) and $Q_{1}, Q_{2}$ as in (2.6) or (2.7). Now we are in conditions to write the reducible theorem.

Theorem 1 Let $A \in \mathbb{R}^{n \times n}$ be an $l \times l$ block matrix with each block of order $k$ and $P$ be defined in (2.8). Then $A \in \mathcal{G}_{l}$ if and only if

$$
P^{T} A P=\left[\begin{array}{ll|ll}
A_{1} & & & \\
& A_{2} & & \\
\hline & & A_{3} & \\
& & & A_{4}
\end{array}\right] .
$$

Proof. $(\Longrightarrow)$ Since $A \in \mathcal{B}_{l}$, we have, from Corollary $1, Q^{T} A Q=\operatorname{diag}(M, N)$. Because $A \in \mathcal{W}_{l}$ it results from (2.4) and (2.5) that $M, N \in \mathcal{W}_{l}$. Applying Corollary 2 to each one of $M$ and $N$ gives $Q_{1}^{T} M Q_{1}=\operatorname{diag}\left(A_{1}, A_{2}\right)$ and $Q_{2}^{T} N Q_{2}=\operatorname{diag}\left(A_{3}, A_{4}\right)$ and the decomposition (2.9) follows.

The proof of the converse is similar.

\subsection{Eigenstructure of generalized centrosymmetric matrices}

In this subsection we investigate the eigenstructure of generalized centrosymmetric matrices. All the formulae become slightly more complicated when $l$ or $k$ is odd; for simplicity, in the remainder of this paper we will restrict our attention to the even case, i.e., when $l=2 s$ and $k=2 t$.

Definition 3 Let $\mathbf{x} \in \mathbb{R}^{n}$ be an l-block vector with each block of dimension $k$. Vector $\mathbf{x}$ is said to be l-block symmetric if $\Pi_{l, k} \mathbf{x}=\mathbf{x}, l$-block skew symmetric if $\Pi_{l, k} \mathbf{x}=-\mathbf{x}, l$-block-wise symmetric if $\Theta_{l, k} \mathbf{x}=\mathbf{x}$ and $l$-block-wise skew symmetric if $\Theta_{l, k} \mathbf{x}=-\mathbf{x}$.

An $l$-block symmetric vector $\mathbf{x} \in \mathbb{R}^{n}$ has the form

$$
\mathbf{x}=\left[\begin{array}{llllll}
\mathbf{x}_{1}^{T} & \ldots & \mathbf{x}_{s}^{T} & \mathbf{x}_{s}^{T} & \ldots & \mathbf{x}_{1}^{T}
\end{array}\right]^{T}
$$

and an $l$-block skew symmetric vector $\hat{\mathbf{x}} \in \mathbb{R}^{n}$ is of the form

$$
\hat{\mathbf{x}}=\left[\begin{array}{llllll}
\hat{\mathbf{x}}_{1}^{T} & \ldots & \hat{\mathbf{x}}_{s}^{T} & -\hat{\mathbf{x}}_{s}^{T} & \ldots & -\hat{\mathbf{x}}_{1}^{T}
\end{array}\right]^{T}
$$

where $\mathbf{x}_{i}, \hat{\mathbf{x}}_{i} \in \mathbb{R}^{k}$. So we can write an $l$-block symmetric vector $\mathbf{x} \in \mathbb{R}^{n}$ and an $l$-block skew symmetric vector $\hat{\mathbf{x}} \in \mathbb{R}^{n}$ as follows:

$$
\mathbf{x}=\left[\begin{array}{c}
\mathbf{y} \\
\Pi_{s, k} \mathbf{y}
\end{array}\right] \quad \text { where } \quad \mathbf{y}=\left[\begin{array}{c}
\mathbf{x}_{1} \\
\vdots \\
\mathbf{x}_{s}
\end{array}\right] \in \mathbb{R}^{n / 2}
$$


and

$$
\hat{\mathbf{x}}=\left[\begin{array}{c}
\hat{\mathbf{y}}_{s, k} \hat{\mathbf{y}}
\end{array}\right] \quad \text { where } \quad \hat{\mathbf{y}}=\left[\begin{array}{c}
\hat{\mathbf{x}}_{1} \\
\vdots \\
\hat{\mathbf{x}}_{s}
\end{array}\right] \in \mathbb{R}^{n / 2} .
$$

Similarly, an $l$-block-wise symmetric vector $\mathbf{x} \in \mathbb{R}^{n}$ has the form

$$
\tilde{\mathbf{x}}=\left[\begin{array}{lllll}
\tilde{\mathbf{x}}_{1}^{T} & \left(J_{t} \tilde{\mathbf{x}}_{1}\right)^{T} & \ldots & \tilde{\mathbf{x}}_{l}^{T} & \left(J_{t} \tilde{\mathbf{x}}_{l}\right)^{T}
\end{array}\right]^{T}, \quad \tilde{\mathbf{x}}_{i} \in \mathbb{R}^{t} .
$$

An $l$-block-wise skew symmetric vector $\breve{\mathbf{x}} \in \mathbb{R}^{n}$ has the form

$$
\breve{\mathbf{x}}=\left[\begin{array}{lllll}
\breve{\mathbf{x}}_{1}^{T} & -\left(J_{t} \breve{\mathbf{x}}_{1}\right)^{T} & \ldots & \breve{\mathbf{x}}_{l}^{T} & -\left(J_{t} \breve{\mathbf{x}}_{l}\right)^{T}
\end{array}\right]^{T}, \quad \breve{\mathbf{x}}_{i} \in \mathbb{R}^{t} .
$$

The following lemma derives from straightforward calculations.

Lemma 3 Let matrices $Q$ and $\hat{Q}$ be defined in (2.3) and (2.6), respectively.

(i) If $\mathbf{x} \in \mathbb{R}^{n}$ is l-block symmetric, then

$$
Q^{T} \mathbf{x}=\left[\begin{array}{c}
\mathbf{z}_{1} \\
0
\end{array}\right], \quad \text { where } \quad \mathbf{z}_{1}=\sqrt{2} \mathbf{y} \quad \text { with } \quad \mathbf{y} \text { given in }(2.10) .
$$

(ii) If $\hat{\mathbf{x}} \in \mathbb{R}^{n}$ is l-block skew symmetric, then

$$
Q^{T} \hat{\mathbf{x}}=\left[\begin{array}{c}
0 \\
\mathbf{z}_{2}
\end{array}\right], \quad \text { where } \quad \mathbf{z}_{2}=\sqrt{2} \hat{\mathbf{y}} \quad \text { with } \hat{\mathbf{y}} \text { given in }(2.11) .
$$

(iii) If $\tilde{\mathbf{x}} \in \mathbb{R}^{n}$ is l-block-wise symmetric, then

$$
\hat{Q}^{T} \tilde{\mathbf{x}}=\left[\begin{array}{c}
\mathbf{u} \\
0
\end{array}\right], \quad \text { where } \mathbf{u}=\sqrt{\mathbf{2}}\left[\begin{array}{c}
\tilde{\mathbf{x}}_{1} \\
\vdots \\
\tilde{\mathbf{x}}_{l}
\end{array}\right] .
$$

(iv) If $\breve{\mathbf{x}} \in \mathbb{R}^{n}$ is l-block-wise skew symmetric, then

$$
\hat{Q}^{T} \breve{\mathbf{x}}=\left[\begin{array}{c}
0 \\
\mathbf{v}
\end{array}\right], \quad \text { where } \mathbf{v}=\sqrt{\mathbf{2}}\left[\begin{array}{c}
\breve{\mathbf{x}}_{1} \\
\vdots \\
\breve{\mathbf{x}}_{l}
\end{array}\right] .
$$

Lemma 4 Let $A \in \mathbb{R}^{n \times n}$ be an $l \times l$ block matrix with each block of order $k$. We have the following statements:

(i) If $A \in \mathcal{B}_{l}$, then the eigenvectors of $A$ are either l-block symmetric or l-block skew symmetric.

(ii) If $A \in \mathcal{W}_{l}$, then the eigenvectors of $A$ are either l-block-wise symmetric or l-block-wise skew symmetric.

Proof. We first prove statement (i). Assume that $\lambda$ is an eigenvalue of $A$ and $\mathbf{y}$ is the corresponding eigenvector. Since to $\Pi_{l, k} A \Pi_{l, k}=A$ and $\Pi_{l, k}^{2}=I$, we have

$$
A \Pi_{l, k} \mathbf{y}=\lambda \Pi_{l, k} \mathbf{y} .
$$

From the equalities above, we obtain

$$
A \frac{I+\Pi_{l, k}}{2} \mathbf{y}=\lambda \frac{I+\Pi_{l, k}}{2} \mathbf{y} \quad \text { and } \quad A \frac{I-\Pi_{l, k}}{2} \mathbf{y}=\lambda \frac{I-\Pi_{l, k}}{2} \mathbf{y} .
$$

It is easy to see that $\frac{I+\Pi_{l, k}}{2} \mathbf{y}$ is $l$-block symmetric and $\frac{I-\Pi_{l, k}}{2} \mathbf{y}$ is $l$-block skew symmetric. Based on the above facts, we have that the eigenvector of $A$ associated with the eigenvalue $\lambda$ is either $l$-block symmetric or $l$-block skew symmetric. 
Using the fact that $\Theta_{l, k} A \Theta_{l, k}=A$ and $\Theta_{l, k}^{2}=I$, a similar argument shows that statement (ii) holds.

We are now able to characterize the eigenstructure of a real block symmetric matrix $A \in \mathcal{G}_{l}$, which can be derived from Theorem 1 .

Corollary 3 Let $A \in \mathcal{G}_{l}$ be real block symmetric, $P$ defined in (2.8) and $X$ the matrix of orthonormal eigenvectors of $A$. Then

$$
X=P Y \quad \text { with } Y=\operatorname{diag}\left(Y_{1}, Y_{2}, Y_{3}, Y_{4}\right)
$$

where $Y_{1}, Y_{2}, Y_{3}$ and $Y_{4}$ are orthogonal matrices whose columns are the eigenvectors of $A_{1}, A_{2}, A_{3}$ and $A_{4}$ defined in (2.9), respectively.

From this result we can verify that for any $A \in \mathcal{G}_{l}$ there exist $n / 4 l$-block symmetric and $l$-block-wise symmetric eigenvectors, $n / 4 l$-block symmetric and $l$ block-wise skew symmetric eigenvectors, $n / 4 l$-block skew symmetric and $l$-blockwise symmetric eigenvectors, and $n / 4 l$-block skew symmetric and $l$-block-wise skew symmetric eigenvectors.

Recalling the definitions of even and odd eigenvalues of centrosymmetric matrices, we give the following definition for a matrix $A \in \mathcal{G}_{l}$.

Definition 4 Let $A \in \mathcal{G}_{l}$ and $\lambda$ be an eigenvalue of $A$. Then $\lambda$ is said to be eveneven, even-odd, odd-even or odd-odd, if $\lambda$ is associated with an l-block symmetric and l-block-wise symmetric, l-block symmetric and l-block-wise skew symmetric, $l$ block skew symmetric and l-block-wise symmetric, or l-block skew symmetric and l-block-wise skew symmetric eigenvector.

Theorem 2 Given an arbitrary real diagonal matrix $\Lambda=\operatorname{diag}\left(\lambda_{1}, \ldots, \lambda_{n}\right)$ and orthogonal matrix $Y$ defined in (2.12), we have

$$
A=P Y \Lambda Y^{T} P^{T} \in \mathcal{G}_{l} .
$$

In this case, $\left\{\lambda_{i}\right\}_{i=1}^{n / 4}$ are the eigenvalues of $A$ with even-even pattern, $\left\{\lambda_{i}\right\}_{i=n / 4+1}^{n / 2}$ the eigenvalues with even-odd pattern, $\left\{\lambda_{i}\right\}_{i=n / 2+1}^{3 n / 4}$ the eigenvalues with odd-even pattern, and $\left\{\lambda_{i}\right\}_{i=3 n / 4+1}^{n}$ the eigenvalues with odd-odd pattern.

\section{Structure-preserving algorithm}

The methods presented in [12] do not produce a RSBT matrix but the ideas therein may be combined with the structure of the eigenvectors studied in the previous section to solve our Problem 1. A major decision to be made is how to partition the target spectrum in 4 subsets which do correspond to the 4 blocks in the decomposition (2.9). In fact, as it may be seen in the examples presented in section 4, some partitions may lead to convergence whereas others fail. So, the solutions of Problem 1 are the solutions of the following problems, one for each partition of the spectrum.

Problem 2 Given real numbers $\tau_{1}^{*} \leq \ldots \leq \tau_{n / 4}^{*}, \mu_{1}^{*} \leq \ldots \leq \mu_{n / 4}^{*}, \nu_{1}^{*} \leq \ldots \leq \nu_{n / 4}^{*}$, $\omega_{1}^{*} \leq \ldots \leq \omega_{n / 4}^{*}$ and $l \in \mathbb{N}$, find $\mathbf{c} \in \mathbb{R}^{n}$ such that $T(\mathbf{c})$ is an $l \times l$ RSBT matrix with symmetric Toeplitz blocks and

$$
\tau_{i}(\mathbf{c})=\tau_{i}^{*}, \quad \mu_{i}(\mathbf{c})=\mu_{i}^{*} \quad \nu_{i}(\mathbf{c})=\nu_{i}^{*}, \quad \omega_{i}(\mathbf{c})=\omega_{i}^{*}, \quad i=1, \cdots, n / 4,
$$

where $\left\{\tau_{i}(\mathbf{c})\right\} \cup\left\{\mu_{i}(\mathbf{c})\right\} \cup\left\{\nu_{i}(\mathbf{c})\right\} \cup\left\{\omega_{i}(\mathbf{c})\right\}$ are the eigenvalues of $T(\mathbf{c})$. 


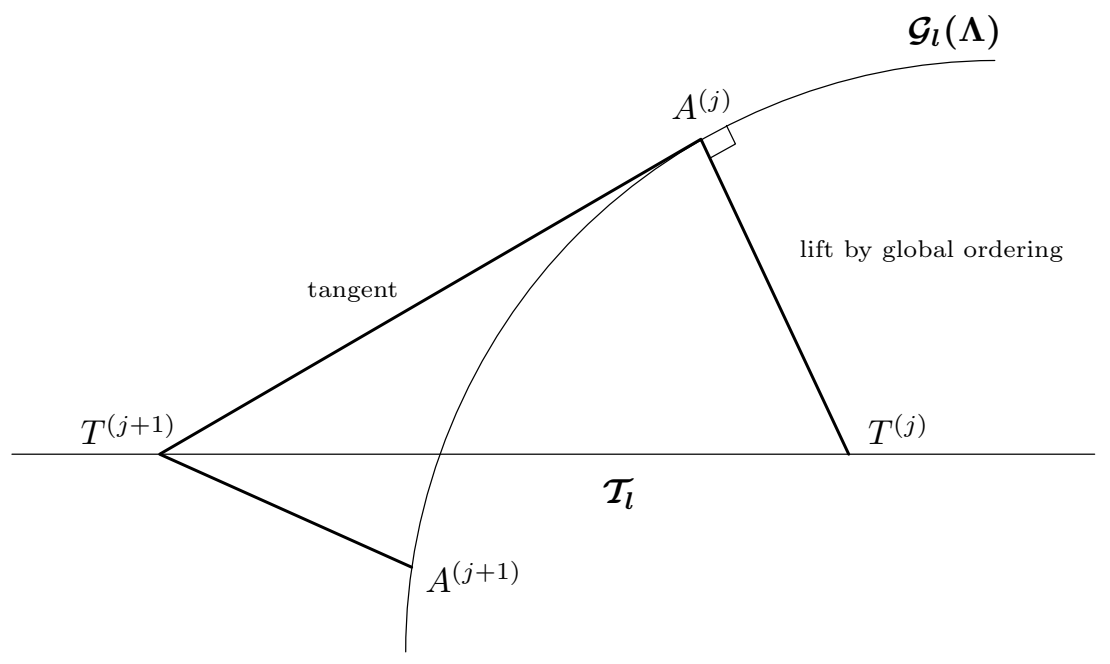

Figure 1: Geometry of lift by Wielandt-Hoffman Theorem

We call $\tau_{i}^{*}, \mu_{i}^{*}, \nu_{i}^{*}$ and $\omega_{i}^{*}, i=1, \ldots, n / 4$, the even-even, even-odd, oddeven and odd-odd target eigenvalues, respectively, and $\Sigma_{1}^{*}=\left\{\tau_{1}^{*}, \ldots, \tau_{n / 4}^{*}\right\}, \Sigma_{2}^{*}=$ $\left\{\mu_{1}^{*}, \ldots, \mu_{n / 4}^{*}\right\}, \Sigma_{3}^{*}=\left\{\nu_{1}^{*}, \ldots, \nu_{n / 4}^{*}\right\}$ and $\Sigma_{4}^{*}=\left\{\omega_{1}^{*}, \ldots, \omega_{n / 4}^{*}\right\}$ the even-even, even-odd, odd-even and odd-odd target spectrum, respectively. Also, we define $\Sigma_{1}=\operatorname{diag}\left(\tau_{1}^{*}, \ldots, \tau_{n / 4}^{*}\right), \Sigma_{2}=\operatorname{diag}\left(\mu_{1}^{*}, \ldots, \mu_{n / 4}^{*}\right), \Sigma_{3}=\operatorname{diag}\left(\nu_{1}^{*}, \ldots, \nu_{n / 4}^{*}\right), \Sigma_{4}=$ $\operatorname{diag}\left(\omega_{1}^{*}, \ldots, \omega_{n / 4}^{*}\right)$ and $\Lambda=\operatorname{diag}\left(\Sigma_{1}, \Sigma_{2}, \Sigma_{3}, \Sigma_{4}\right)$.

The basic ideas we follow to develop an eficient algorithm for Problem 2 are described in $[5,6,8]$. We will briefly resume the underlying theory.

The classical Newton iteration

$$
x^{(j+1)}=x^{(j)}-\left(f^{\prime}\left(x^{(j)}\right)\right)^{-1} f\left(x^{(j)}\right)
$$

for finding a zero of a scalar function $f: \mathbb{R} \rightarrow \mathbb{R}$ can be thought as a two-step procedure: the tangent step, with $x^{(j+1)}$ being the $x$-intercept of the tangent line to the graph of $f$ in $\left(x^{(j)}, f\left(x^{(j)}\right)\right)$, and the lift step where the point $\left(x^{(j+1)}, f\left(x^{(j+1)}\right)\right)$ is a natural lift of the $x$-intercept along the $y$-axis to the graph of $f$. An analogue of this idea for the IEP for RSBT matrices with symmetric Toeplitz blocks is to think of the isospectral subset $\mathcal{G}_{l}=\mathcal{G}_{l}(\Lambda)$ of generalized $K$-centrosymmetric matrices as the graph of some unknown $f$, and the subspace $\mathcal{T}_{l}$ of $l \times l$ RSBT matrices with symmetric Toeplitz blocks as the $x$-axis. All we have to do is to perform the tangent and lift steps with the subspaces $\mathcal{G}_{l}$ and $\mathcal{T}_{l}$. See figure 1 .

By Theorem 2, we know that every matrix $A \in \mathcal{G}_{l}$ may be written as $A=X \Lambda X^{T}$ with $X=P Y$ defined in (2.12). It can be shown that the tangent vectors of $\mathcal{G}_{l}$ at $A$ are given by

$$
\mathcal{K}_{A}\left(\mathcal{G}_{l}\right)=\tilde{L} A-A \tilde{L},
$$

where $\tilde{L}=X L X^{T}$, with $L=\operatorname{diag}\left(L_{1}, L_{2}, L_{3}, L_{4}\right)$ and $L_{i} \in \mathbb{R}^{\frac{n}{4} \times \frac{n}{4}}, i=1, \ldots, 4$, being skew symmetric. Thus, a tangent step from a given $A^{(j)} \in \mathcal{G}_{l}(\Lambda)$ is equivalent to find a skew symmetric matrix $\tilde{L}$ and a vector $\mathbf{c}^{(j+1)}$ such that $T^{(j+1)}=T\left(\mathbf{c}^{(j+1)}\right)$ satisfies

$$
A^{(j)}+\tilde{L}^{(j)} A^{(j)}-A^{(j)} \tilde{L}^{(j)}=T^{(j+1)} .
$$

Since $A^{(j)} \in \mathcal{G}_{l}(\Lambda)$, by Theorem 2, there exists an orthogonal matrix $X^{(j)}$ such that $A^{(j)}=X^{(j)} \Lambda X^{(j)^{T}}$ with $X^{(j)}=P Y^{(j)}$ given by (2.12). Also, we obtain 
$Y^{(j)}=\operatorname{diag}\left(Y_{1}^{(j)}, Y_{2}^{(j)}, Y_{3}^{(j)}, Y_{4}^{(j)}\right)$.

Left-multiplying by $X^{(j)^{T}}$ and right-multiplying by $X^{(j)}$ equation (3.15), we have

$$
\Lambda+L^{(j)} \Lambda-\Lambda L^{(j)}=Y^{(j)^{T}}\left[P^{T} T^{(j+1)} P\right] Y^{(j)},
$$

where $L^{(j)}=X^{(j)^{T}} \tilde{L}^{(j)} X^{(j)}=\operatorname{diag}\left(L_{1}^{(j)}, L_{2}^{(j)}, L_{3}^{(j)}, L_{4}^{(j)}\right)$ is also skew symmetric.

Notice that, since since $T^{(j+1)} \in \mathcal{G}_{l}$, by Theorem $1, P^{T} T^{(j+1)} P$ is a $4 \times 4$ diagonal block matrix,

$$
P^{T} T^{(j+1)} P=\operatorname{diag}\left(\tilde{T}_{1}^{(j+1)}, \tilde{T}_{2}^{(j+1)}, \tilde{T}_{3}^{(j+1)}, \tilde{T}_{4}^{(j+1)}\right) .
$$

Thus equation (3.16) breaks down into four disjoint blocks,

$$
\Sigma_{i}+L_{i}^{(j)} \Sigma_{i}-\Sigma_{i} L_{i}^{(j)}=Y_{i}^{(j)^{T}} \tilde{T}_{i}^{(j+1)} Y_{i}^{(j)}, \quad i=1, \ldots, 4 .
$$

Notice now that $T^{(j+1)}=\sum_{i=1}^{n} c_{i}^{(j+1)} S_{i}$ with $S_{i} \in \mathcal{G}_{l}$. It follows from Theorem 1 that

$$
P^{T} S_{i} P=\operatorname{diag}\left(S_{i}^{[1]}, S_{i}^{[2]}, S_{i}^{[3]}, S_{i}^{[4]}\right)
$$

and thus

$$
\begin{aligned}
& Y^{(j)^{T}}\left[P^{T} T\left(\mathbf{c}^{(j+1)}\right) P\right] Y^{(j)}= \\
& \sum_{i=1}^{n} c_{i}^{(j+1)} \operatorname{diag}\left(Y_{1}^{(j)^{T}} S_{i}^{[1]} Y_{1}^{(j)}, Y_{2}^{(j)^{T}} S_{i}^{[2]} Y_{2}^{(j)}, Y_{3}^{(j)^{T}} S_{i}^{[3]} Y_{3}^{(j)}, Y_{4}^{(j)^{T}} S_{i}^{[4]} Y_{4}^{(j)}\right),
\end{aligned}
$$

where $S_{i}^{[1]}, S_{i}^{[2]}, S_{i}^{[3]}$ and $S_{i}^{[4]} \in \mathbb{R}^{\frac{n}{4} \times \frac{n}{4}}$ are constant matrices for $i=1, \ldots, n$.

Comparing the $n$ diagonal entries on both sides of (3.16), we obtain a linear system for $\mathbf{c}^{(j+1)}$,

$$
\Gamma^{(j)} \mathbf{c}^{(j+1)}=\mathbf{b},
$$

where $\mathbf{b}=\left[\Sigma_{1}^{*}, \Sigma_{2}^{*}, \Sigma_{3}^{*}, \Sigma_{4}^{*}\right]^{T}$ and $\Gamma^{(j)}=\left[\gamma_{p q}^{(j)}\right]_{p, q=1, \ldots, n}$ with

$$
\gamma_{p i}^{(j)}= \begin{cases}\left(Y_{1:, p]}^{(j)}\right)^{T} S_{i}^{[1]}\left(Y_{1}^{(j)}\right)_{[:, p]}, & \text { if } \quad 1 \leq p \leq \frac{n}{4} \\ \left(Y_{[:, p]}^{(j)}\right)^{T} S_{i}^{[2]}\left(Y_{2}^{(j)}\right)_{[:, p]}, & \text { if } \quad \frac{n}{4}+1 \leq p \leq \frac{n}{2} \\ \left(Y_{3}^{(j)}{ }_{[:, p]}\right)^{T} S_{i}^{[3]}\left(Y_{3}^{(j)}\right)_{[:, p]}, & \text { if } \quad \frac{n}{2}+1 \leq p \leq \frac{3 n}{4} \\ \left(Y_{4:[;, p]}^{(j)}\right)^{T} S_{i}^{[4]}\left(Y_{4}^{(j)}\right)_{[:, p]}, & \text { if } \quad \frac{3 n}{4}+1 \leq p \leq n\end{cases}
$$

for $i=1, \ldots, n$, with $\left(Y_{q}^{(j)}\right)_{[:, p]}$ denoting the $p^{t h}$ column of the matrix $Y_{q}^{(j)}$.

To form $\Gamma^{(j)}$, we need only to do matrix-vector and vector-vector products in (3.20) of lenght about $n / 4$. By solving (3.19), we then get the vector $\mathbf{c}^{(j+1)}$, and thus the matrix $T^{(j+1)}$.

The next task is to lift the matrix $T^{(j+1)} \in \mathcal{T}_{l}$ back to $\mathcal{G}_{l}(\Lambda)$. There are several known ways to do that.

One well-known way to perform the lift step is to compute the approximation based on the use of matrix exponentials and Cayley transforms. Suppose that all iterations are taking place near a point of intersection of the two sets $\mathcal{G}_{l}(\Lambda)$ and $\mathcal{T}_{l}$. Then we should have

$$
A^{(j+1)} \approx T^{(j+1)}
$$


and, from (3.16), we also have

$$
T^{(j+1)} \approx e^{-\tilde{L}^{(j)}} A^{(j)} e^{\tilde{L}^{(j)}} .
$$

It is very expensive to compute $e^{\tilde{L}^{(j)}}$ and it is only an approximation. Instead, the Cayley transform gives

$$
\tilde{Z}^{(j)}=\left(I+\frac{\tilde{L}^{(j)}}{2}\right)\left(I-\frac{\tilde{L}^{(j)}}{2}\right)^{-1},
$$

which is an orthogonal matrix and the $(1,1)$ Padé approximation of $e^{\tilde{L}^{(j)}}$. Thus, we have

$$
\tilde{Z}^{(j)} \approx e^{\tilde{L}^{(j)}} .
$$

Now we can define

$$
A^{(j+1)}=\left(\tilde{Z}^{(j)}\right)^{T} A^{(j)} \tilde{Z}^{(j)}=\left(\tilde{Z}^{(j)}\right)^{T} X^{(j)} \Lambda X^{(j)^{T}} \tilde{Z}^{(j)} \in \mathcal{G}_{l}
$$

assuming that $\left\|\tilde{L}^{(j)}\right\|$ is small. Defining $X^{(j+1)}=\left(\tilde{Z}^{(j)}\right)^{T} X^{(j)}$ gives

$$
A^{(j+1)}=X^{(j+1)} \Lambda X^{(j+1)^{T}}
$$

which is the spectral decomposition of $A^{(j+1)}$ for the next tangent step. In practice the lift matrix $A^{(j+1)}$ needs not to be formed explicitly. Given (3.16), we only need to compute the orthogonal matrix using

$$
Y^{(j+1)}=Y^{(j)} Z^{(j)^{T}}
$$

where

$$
Z^{(j)}=\left(I+\frac{L^{(j)}}{2}\right)\left(I-\frac{L^{(j)}}{2}\right)^{-1}
$$

In $(3.22)$ we used the fact that $X^{(j)^{T}}\left(\tilde{Z}^{(j)}\right)^{T} X^{(j)}=Z^{(j)^{T}}$, where $Z^{(j)}=\operatorname{diag}\left(Z_{1}^{(j)}, Z_{2}^{(j)}, Z_{3}^{(j)}, Z_{4}^{(j)}\right)$. Observe that to obtain $Z^{(j)}$ in (3.23), we also need to compute $L^{(j)}$. This can be done by comparing the off-diagonal entries on both sides of (3.16) or (3.17), provided that $T^{(j+1)}$ is determined and the scalars in each of the groups $\left\{\tau_{i}^{*}\right\}_{i=1}^{n / 4},\left\{\mu_{i}^{*}\right\}_{i=1}^{n / 4},\left\{\nu_{i}^{*}\right\}_{i=1}^{n / 4}$ and $\left\{\omega_{i}^{*}\right\}_{i=1}^{n / 4}$ are distinct. The formulae are the following:

$$
\begin{aligned}
& \left(L_{1}^{(j)}\right)_{p q}=\frac{\left(Y_{1}^{(j)}{ }_{[:, p]}\right)^{T} \tilde{T}_{1}^{(j+1)}\left(Y_{1}^{(j)}{ }_{[:, q]}\right)}{\tau_{q}^{*}-\tau_{p}^{*}}, \quad\left(L_{2}^{(j)}\right)_{p q}=\frac{\left(Y_{2}^{(j)}{ }_{[i, p]}\right)^{T} \tilde{T}_{2}^{(j+1)}\left(Y_{2}^{(j)}{ }_{[:, q]}\right)}{\mu_{q}^{*}-\mu_{p}^{*}}, \\
& \left(L_{3}^{(j)}\right)_{p q}=\frac{\left(Y_{3}^{(j)}{ }_{[:, p]}\right)^{T} \tilde{T}_{3}^{(j+1)}\left(Y_{3}^{(j)}{ }_{[:, q]}\right)}{\nu_{q}^{*}-\nu_{p}^{*}}, \quad\left(L_{4}^{(j)}\right)_{p q}=\frac{\left(Y_{4}^{(j)}{ }_{[:, p]}\right)^{T} \tilde{T}_{4}^{(j+1)}\left(Y_{4}^{(j)}{ }_{[:, q]}\right)}{\omega_{q}^{*}-\omega_{p}^{*}},
\end{aligned}
$$

for $1 \leq p<q \leq \frac{n}{4}$.

In practice, to compute $\left(Z^{(j)}\right)^{T}$ we avoid the computation of the inverse in (3.23). If we let

$$
B^{(j)}=\left(I+\frac{L^{(j)}}{2}\right) \text { and } C^{(j)}=I-\frac{L^{(j)}}{2}
$$


then we can write

$$
Z^{(j)} C^{(j)}=B^{(j)}
$$

Since $L^{(j)}$ is skewsymmetric, we have $\left(C^{(j)}\right)^{T}=B^{(j)}$ and then

$$
B^{(j)}\left(Z^{(j)}\right)^{T}=\left(B^{(j)}\right)^{T} \text {. }
$$

Thus, from

$$
\begin{aligned}
B^{(j)} & =\operatorname{diag}\left(I+\frac{L_{1}^{(j)}}{2}, I+\frac{L_{2}^{(j)}}{2}, I+\frac{L_{3}^{(j)}}{2}, I+\frac{L_{4}^{(j)}}{2}\right) \\
& =\operatorname{diag}\left(B_{1}^{(j)}, B_{2}^{(j)}, B_{3}^{(j)}, B_{4}^{(j)}\right),
\end{aligned}
$$

we can compute $\left(Z^{(j)}\right)^{T}=\operatorname{diag}\left(Z_{1}^{(j)}, Z_{2}^{(j)}, Z_{3}^{(j)}, Z_{4}^{(j)}\right)^{T}$, by solving

$$
B_{i}^{(j)}\left(Z_{i}^{(j)}\right)^{T}=\left(B_{i}^{(j)}\right)^{T}, \quad i=1, \ldots, 4 .
$$

Thus, a complete Newton iteration involving a tangent step and a lift step between $\mathcal{G}_{l}(\Lambda)$ and $\mathcal{T}_{l}$ has been well built up.

As we have already mentioned, we can regard Problem 2 as a general PIEP and the existing numerical methods in $[5,12]$ can be adapted for this problem. Because the tangent step and the lift step take place in a smaller space $\mathcal{C}_{n}$ (see Lemma 2), $\mathcal{B}_{l}$ or $\mathcal{W}_{l}$, then, analogously to what is used in $[5,8,25]$, one can easily develop a procedure being capable of dealing with the case of double eigenvalues. Such eigenvalues have to be split into two groups, where each group consists of mutually distinct eigenvalues. (see Corollary 1, Corollary 2, Lemma 3 and Lemma 4). Compared to the schemes mentioned above, our structure-preserving technique is able to handle the case of repeated eigenvalues with multiplicity less than or equal to four. Morover, the computational costs of each iteration in our structurepreserving scheme is about $\frac{1}{16}$ of the one in the first scheme and about $\frac{1}{4}$ of the one in the second scheme discussed above. That is to say that our structure-preserving scheme ensure significant savings in computational costs.

On the other hand, the lift will fail or suffer from the numerical instability when the skew symmetric matrix $L_{i}$ can not be defined by (3.24) or $\left\|L_{i}\right\|$ is not small enough. This is the case when repeated eigenvalues or close eigenvalues appear in the same subset.

An alternative method that passes over the formulation of $L_{i}$ in (3.24) and thus avoid the cases mentioned above is to look for a matrix $A^{(j+1)} \in \mathcal{G}_{l}$ that is nearest to $T^{(j+1)}$. Such a nearest approximation can be obtained by the Wielandt-Hoffman theorem. That is, suppose that the spectral decomposition of $T^{(j+1)}$ is

$$
T^{(j+1)}=P \tilde{Y}^{(j+1)} \operatorname{diag}\left(\tilde{\Sigma}_{1}, \tilde{\Sigma}_{2}, \tilde{\Sigma}_{3}, \tilde{\Sigma}_{4}\right)\left(\tilde{Y}^{(j+1)}\right)^{T} P^{T}
$$

where

$$
\tilde{Y}^{(j+1)}=\operatorname{diag}\left(\tilde{Y}_{1}^{(j+1)}, \tilde{Y}_{2}^{(j+1)}, \tilde{Y}_{3}^{(j+1)}, \tilde{Y}_{4}^{(j+1)}\right),
$$

$\tilde{\Sigma}_{1}=\operatorname{diag}\left(\tilde{\tau}_{1}, \ldots, \tilde{\tau}_{n / 4}\right), \tilde{\Sigma}_{2}=\operatorname{diag}\left(\tilde{\mu}_{1}, \ldots, \tilde{\mu}_{n / 4}\right), \tilde{\Sigma}_{3}=\operatorname{diag}\left(\tilde{\nu}_{1}, \ldots, \tilde{\nu}_{n / 4}\right)$ and $\tilde{\Sigma}_{4}=\operatorname{diag}\left(\tilde{\omega}_{1}, \ldots, \tilde{\omega}_{n / 4}\right)$. By permuting the columns of $\tilde{Y}_{i}^{(j+1)}, i=1,2,3,4$, such that the diagonal entries in the corresponding $\tilde{\Sigma}_{1}, \tilde{\Sigma}_{2}, \tilde{\Sigma}_{3}$ and $\tilde{\Sigma}_{4}$ are in the same ordering as those in $\Sigma_{1}, \Sigma_{2}, \Sigma_{3}$, and $\Sigma_{4}$, respectively, we have

$$
T^{(j+1)}=P Y^{(j+1)} \operatorname{diag}\left(\hat{\Sigma}_{1}, \hat{\Sigma}_{2}, \hat{\Sigma}_{3}, \hat{\Sigma}_{4}\right) Y^{(j+1)^{T}} P^{T}
$$


where $Y^{(j+1)}, \hat{\Sigma}_{1}, \hat{\Sigma}_{2}, \hat{\Sigma}_{3}$ and $\hat{\Sigma}_{4}$ denote the reorganized matrices in the corresponding $\tilde{Y}^{(j+1)}, \tilde{\Sigma}_{1}, \tilde{\Sigma}_{2}, \tilde{\Sigma}_{3}$ and $\tilde{\Sigma}_{4}$, respectively. Then the nearest approximation is given by

$$
A^{(j+1)}=P Y^{(j+1)} \operatorname{diag}\left(\Sigma_{1}, \Sigma_{2}, \Sigma_{3}, \Sigma_{4}\right) Y^{(j+1)^{T}} P^{T} .
$$

Again, the lift matrix $A^{(j+1)}$ needs not to be computed. Given (3.16), we only need the matrix $Y^{(j+1)}$ for the next tangent step.

In practical computation, the target spectrum is usually normalized. See [25]. That is, the normal target spectrum $\Lambda^{*}=\left\{\lambda_{1}^{*}, \ldots, \lambda_{n}^{*}\right\}$ satisfies the following relations

$$
\sum_{i=1}^{n} \lambda_{i}^{*}=0 \text { and } \sum_{i=1}^{n}\left(\lambda_{i}^{*}\right)^{2}=1
$$

For any given target spectrum $\check{\Lambda}=\left\{\check{\lambda}_{1}, \ldots, \check{\lambda}_{n}\right\}$ which does not satisfy (3.25), the process of normalization is as follows. Defining, for $i=1, \ldots, n$,

$$
\lambda_{i}^{*}=\frac{\check{\lambda}_{i}-\bar{\lambda}}{\eta}, \quad \text { with } \quad \bar{\lambda}=\sum_{i=1}^{n} \check{\lambda}_{i} / n \text { and } \eta=\left(\sum_{i=1}^{n}\left(\check{\lambda}_{i}-\bar{\lambda}\right)^{2}\right)^{\frac{1}{2}},
$$

we get a new target spectrum $\Lambda^{*}$ satisfying (3.25). In this case, a solution to Problem 1 for the new target spectrum $\Lambda^{*}$ must be of the form $\mathbf{c}=\left(0, c_{2}, \ldots, c_{n}\right)^{T}$ and $\check{\mathbf{c}}=\left(\bar{\lambda}, \eta c_{2}, \ldots, \eta c_{n}\right)^{T}$ will be a solution the original problem for the given target spectrum $\check{\Lambda}$.

\subsection{The algorithm}

In this section we present the details of the structure-preserving algorithm we have described for solving the inverse eigenvalue problem for an $l \times l$ RSBT matrix $T$ with symmetric Toeplitz blocks.

Given the target spectrum $\Lambda^{*}=\Sigma_{1}{ }^{*} \cup \Sigma_{2}{ }^{*} \cup \Sigma_{3}{ }^{*} \cup \Sigma_{4}{ }^{*}$ where the eigenvalues in $\Sigma_{1}{ }^{*}, \Sigma_{2}{ }^{*}, \Sigma_{3}{ }^{*}$ and $\Sigma_{4}{ }^{*}$ are sorted in ascending order, the order of the block matrix $l$ (even integer), the order of the blocks $k$ (even integer), the initial vector $\mathbf{c}^{(0)}=$ $\left[c_{0}^{(0)}, c_{1}^{(0)}, \ldots, c_{n}^{(0)}\right]$, with $n=l \times k$, and the error tolerance $\varepsilon$, the algorithm delivers, after $j$ iterations, the spectrum of the approximated matrix $T\left(\mathbf{c}^{(j+1)}\right), \Lambda^{(j)}=\Sigma_{1}{ }^{(j)} \cup$ $\Sigma_{2}{ }^{(j)} \cup \Sigma_{3}{ }^{(j)} \cup \Sigma_{4}{ }^{(j)}$ with $\Sigma_{1}^{(j)}=\left\{\tau_{1}^{(j)}, \ldots, \tau_{\frac{n}{4}}^{(j)}\right\}, \Sigma_{2}^{(j)}=\left\{\mu_{1}^{(j)}, \ldots, \mu_{\frac{n}{4}}^{(j)}\right\}, \Sigma_{3}^{(j)}=$ $\left\{\nu_{1}^{(j)}, \ldots, \nu_{\frac{n}{4}}^{(j)}\right\}$ and $\Sigma_{4}^{(j)}=\left\{\omega_{1}^{(j)}, \ldots, \omega_{\frac{n}{4}}^{(j)}\right\}$ sorted in ascending order, and the error

$$
\Delta^{(j)}=\left\{\sum_{i=1}^{\frac{n}{4}}\left[\left(\tau_{i}^{(j)}-\tau_{i}^{*}\right)^{2}+\left(\mu_{i}^{(j)}-\mu_{i}^{*}\right)^{2}+\left(\nu_{i}^{(j)}-\nu_{i}^{*}\right)^{2}+\left(\omega_{i}^{(j)}-\omega_{i}^{*}\right)^{2}\right]\right\}^{\frac{1}{2}} .
$$

\section{Algorithm}

1. For a chosen tolerance $\delta$ (for instance, $\delta=10^{-3}$ ),

$$
\text { if } \tau_{i+1}^{*}-\tau_{i}^{*}>\delta, \quad \mu_{i+1}^{*}-\mu_{i}^{*}>\delta, \quad \nu_{i+1}^{*}-\nu_{i}^{*}>\delta \text { and } \omega_{i+1}^{*}-\omega_{i}^{*}>\delta
$$

then choose method 1 (distint target eigenvalues)

else choose method 2 (close target eigenvalues)

2. Compute the orthogonal matrix $P$ accordingly to (2.3) and (2.6), and compute the elements $S_{i}$ of the basis factorized such that

$$
P^{T} S_{i} P=\operatorname{diag}\left(S_{i}^{[1]}, S_{i}^{[2]}, S_{i}^{[3]}, S_{i}^{[4]}\right), \quad i=1, \ldots, n ;
$$


compute $\widetilde{T}_{i}^{(0)}, i=1, \ldots, 4$, such that

$$
\begin{aligned}
P^{T} T\left(\mathbf{c}^{(0)}\right) P & =\operatorname{diag}\left(\widetilde{T}_{1}^{(0)}, \widetilde{T}_{2}^{(0)}, \widetilde{T}_{3}^{(0)}, \widetilde{T}_{4}^{(0)}\right) \\
& =\sum_{i=1}^{n} c_{i}^{(0)} \operatorname{diag}\left(S_{i}^{[1]}, S_{i}^{[2]}, S_{i}^{[3]}, S_{i}^{[4]}\right)
\end{aligned}
$$

and, for each $\widetilde{T}_{i}^{(0)}$, compute the eigenvalues $\Sigma_{i}^{(0)}$ and corresponding eigenvector matrices $Y_{i}^{(0)}$; then sort the eigenvalues in ascending order and the eigenvectors accordingly.

3. Let $j=0, \Delta^{(0)}=1$, num $_{\text {iter }}=0$ and $\max _{i t e r}=200$

while $\Delta^{(j)}>\varepsilon$ and num $_{i t e r}<\max _{i t e r}$

3.1 According to (3.20), solve $\Gamma^{(j)} \mathbf{c}^{(j+1)}=\mathbf{b}$ and compute

$$
\widetilde{T}_{i}^{(j+1)}=\sum_{p=1}^{n} c_{p}^{(j+1)} S_{p}^{[i]}, \quad i=1, \ldots, 4 .
$$

3.2 Compute $\Lambda^{(j+1)}=\Sigma_{1}{ }^{(j+1)} \cup \Sigma_{2}{ }^{(j+1)} \cup \Sigma_{3}{ }^{(j+1)} \cup \Sigma_{4}{ }^{(j+1)}$ where $\Sigma_{i}{ }^{(j+1)}$ is the spectrum of $\widetilde{T}_{i}^{(j+1)}$, respectively (in ascending order).

3.3 Compute the eigenvector matrices $Y_{i}^{(j+1)}, i=1, \ldots, 4$.

For method 1:

3.3.1 Using (3.24), compute the upper triangular elements of $L_{i}^{(j)}$, $i=1, \ldots, 4$. Since $L_{i}^{(j)}$ is skewsymmetric, the lower triangular elements satisfy $\left(L_{i}^{(j)}\right)_{q, p}=-\left(L_{i}^{(j)}\right)_{p, q}, 1 \leq p<q \leq \frac{n}{4}$. Compute

$$
L^{(j)}=\operatorname{diag}\left(L_{1}^{(j)}, L_{2}^{(j)}, L_{3}^{(j)}, L_{4}^{(j)}\right) .
$$

3.3.2 According to (3.23), compute

$$
\left(Z^{(j)}\right)^{T}=\operatorname{diag}\left(Z_{1}^{(j)}, Z_{2}^{(j)}, Z_{3}^{(j)}, Z_{4}^{(j)}\right)^{T},
$$

by solving, for $B_{i}^{(j)}=I+\frac{L_{i}^{(j)}}{2}$, the $\frac{n}{4}$ linear systems

$$
B_{i}^{(j)}\left(Z_{i}^{(j)}\right)_{[:, p]}^{T}=\left(B_{i}^{(j)}\right)_{[;, p]}^{T}, \quad p=1, \ldots, \frac{n}{4} .
$$

3.3.3 Finally, compute

$$
Y_{i}^{(j+1)}=Y_{i}^{(j)}\left(Z_{i}^{(j)}\right)^{T}, \quad i=1, \ldots, 4 .
$$

For method 2:

[Lift by local ordering]

Compute the eigenvector matrix of $\widetilde{T}_{i}^{(j+1)}$ and sort the eigenvectors accordingly to the order of the eigenvalues in $\Sigma_{i}{ }^{(j+1)}$. Let $Y_{i}^{(j+1)}$ be this matrix of eigenvectors.

3.5 Compute the error $\Delta^{(j+1)}$ and increment $j$ and numiter.

\section{end while}

The final matrix is $T\left(\mathbf{c}^{(\mathbf{j})}\right)=P \operatorname{diag}\left(\widetilde{T}_{1}^{(j)}, \widetilde{T}_{2}^{(j)}, \widetilde{T}_{3}^{(j)}, \widetilde{T}_{4}^{(j)}\right) P^{T}$. 


\section{Numerical experiments}

The implementation of the algorithm and all the numerical experiments were carried out with Matlab 7.2.0.232 (R2006a) on a Pentium M 1.6GHz machine under Windows XP. We now present the results of some of our tests in which we have always used $\delta=10^{-3}$. We will use examples for which we know that solutions do exist for Problem 1. In all cases, we iterate until we the error $\Delta^{(j)}$, as defined in (3.26), is smaller than $10^{-10}$ (the arithmetic roundoff error unit is $\epsilon=2^{-53} \approx 10^{-16}$ ).

Example 1 ( $\left.l=2, k=4, \Lambda^{*}=\{1,1,1,1,3,3,3,3\}\right)$. Different solutions (if any) occur for different choices of the $\Sigma_{1}^{*}, \Sigma_{2}^{*}, \Sigma_{3}^{*}$ and $\Sigma_{4}^{*}$. With $\left\{\Sigma_{1}^{*}, \Sigma_{2}^{*}, \Sigma_{3}^{*}, \Sigma_{4}^{*}\right\}=$ $\{\{3,3\},\{3,3\},\{1,1\},\{1,1\}\}$ the exact solution is $T=\left[\begin{array}{cc}2 I & -I \\ -I & 2 I\end{array}\right]$ and just one iteration, independently of the initial vector of parameters $\mathbf{c}^{(0)}$, is enough to produce an approximation $\widetilde{T}$ with $\Delta^{(1)} \approx 7 \times 10^{-16}$ (the maximum difference between any two corresponding entries in $T$ and $\widetilde{T}$ has magnitude $\left.10^{-15}\right)$. A reversal of the order of the previous subsets produces $\widetilde{T} \approx\left[\begin{array}{cc}2 I & I \\ I & 2 I\end{array}\right]$. For $\left\{\Sigma_{1}^{*}, \Sigma_{2}^{*}, \Sigma_{3}^{*}, \Sigma_{4}^{*}\right\}=$ $\{\{1,1\},\{1,3\},\{3,3\},\{1,3\}\}$ an exact solution is

$$
T=\left[\begin{array}{cc}
2 I & S \\
S & 2 I
\end{array}\right] \text { with } S=\left[\begin{array}{cccc}
-1 / 2 & -1 / 2 & 1 / 2 & -1 / 2 \\
-1 / 2 & -1 / 2 & -1 / 2 & 1 / 2 \\
1 / 2 & -1 / 2 & -1 / 2 & -1 / 2 \\
-1 / 2 & 1 / 2 & -1 / 2 & -1 / 2
\end{array}\right]
$$

In this case not every $\mathbf{c}^{(0)}$ delivers convergence. In our trials with random $\mathbf{c}^{(0)}$ we got convergence in about $25 \%$ of the trials and the number of required iterations varies between 2 and 8 . With $\mathbf{c}^{(0)}=\mathbf{e}_{i}$, the ith column of the identity matrix, the method converges in just 1 iteration for $i=7$ (it takes 7 iterations for $i=8$ and diverges for the other columns of the identity). An exchange of the order of the first or the last two subsets in $\{\{1,1\},\{1,3\},\{3,3\},\{1,3\}\}$ produces problems which have solutions $T$ of the same form with $S$ differing from the previous one only in the sign of some diagonals. However, other choices of $\left\{\Sigma_{1}^{*}, \Sigma_{2}^{*}, \Sigma_{3}^{*}, \Sigma_{4}^{*}\right\}$, like $\{\{1,1\},\{3,3\},\{1,3\},\{1,3\}\}$, do not lead to a solution.

Example $2\left(l=4, k=2, \Lambda^{*}=\{1,2,3,4,5,6,7,8\}\right)$. With $\left\{\Sigma_{1}^{*}, \Sigma_{2}^{*}, \Sigma_{3}^{*}, \Sigma_{4}^{*}\right\}=$ $\{\{1,2\},\{3,4\},\{5,6\},\{7,8\}\}$ we could not find a solution in many trials with different $\mathbf{c}^{(0)}$. Interestingly, the matrices we got are not at all random. Different choices of $\mathbf{c}^{(0)}$ produced exactly four different errors only, the smallest of which is $\Delta^{(j)}=0.4364$. We are not in a position to claim that this corresponds to the best possible "solution" of the problem, although we do believe that this is the case. We also tested different orderings $\left\{\Sigma_{1}^{*}, \Sigma_{2}^{*}, \Sigma_{3}^{*}, \Sigma_{4}^{*}\right\}$ and could not get a better result.

Each iteration or our algorithm requires solving a linear system $\Gamma^{(j)} \mathbf{c}^{(j+1)}=\mathbf{b}$. It is not unusual at all for $\Gamma^{(j)}$ to be numerically singular. To prevent early breakdown, in such cases our code uses the least squares approximation.

\section{Conclusions}

We have devised an algorithm for the inverse eigenvalue problem for real symmetric block Toeplitz matrices with symmetric Toeplitz blocks (RSBT). Our computational procedure is based on a Newton type iteration involving a tangent step and a lift step. Such iterative scheme has been used by other authors (mainly by Chu $[5,6,8]$ following the original work of Friedland et al. [12]) for the solution of 
IEP's. The novelty of our work is the application of this scheme to the much more structured RSBT matrices. In our problem, by taking advantage of the structure of the eigenvectors, not only we manage to build up a matrix which has indeed the required strucuture, but we do it more efficiently, as compared to the similar methods that others have used to solve less structured IEP's.

We have developed a Matlab implementation of our algorithm and used the code to try to produce RSBT matrices (of small size, up to 16) for different spectra, including eigenvalues of algebraic multiplicity greater than one. It is important to stress out that our code requires the user to decide how to combine the target eigenvalues to produce an ordered sequence of four sets of $n / 4$ eigenvalues each, corresponding to the four submatrices into which any RSBT matrix may be decomposed through a simple orthogonal similarity transformation. The final matrix, if it exists, depends upon this ordered sequence. Our examples show that some orderings do lead to convergence and others do not. Also, we have experienced that the convergence of the iterative scheme is very sensitive to the initial vector of parameters $\mathbf{c}^{(0)}$ which determines the initial approximation for the RSBT matrix. Interestingly, some column of the identity matrix seems to be, in many cases, a better choice than a random $\mathbf{c}^{(0)}$. In many cases, the code has failed to produce a solution but it may just be the case that such solution does not exist (we do not

have an example where convergence did not occur for a solution which is known to exist). In several of our tests the iterations became stationary at some matrix with spectrum close but not equal to the target spectrum.

\section{References}

[1] A. L. Andrew, Eigenvectors of certain matrices, Linear Alg. Appl., 7: 151-162 (1973).

[2] F. W. Biegler-König, Sufficient conduitions for the solubility of inverse eigenvalue problems, Linear Alg. Appl., 40: 89-100 (1981).

[3] D. A. Bini and B. Meini, Inverting block Toeplitz matrices in block Hessenberg form by means of displacement operators: application to queueing problems, Linear Alg. Appl., 272: 1-16 (1998).

[4] D. Boley, and G.H. Golub, A survey of matrix inverse eigenvalue problems, Inverse Problems, 3: 595-622 (1987).

[5] M. T. Chu, On the refinement of a Newton method for the inverse Toeplitz eigenvalue problem, Available at http://www4.ncsu.edu/ mtchu (1994).

[6] M. T. Chu, Inverse eigenvalue problems, SIAM Rev., 40: 1-39 (1998).

[7] M. T. Chu, F. Diele and S. Ragni, On the inverse problem of constructing symmetric pentadiagonal Toeplitz matrices from their three largest eigenvalues, Inverse Problem, 21: 1879-1894 (2005).

[8] M. T. Chu, and G. H. Golub, Structured inverse eigenvalue problems, Acta Numer., 11: 1-71 (2002).

[9] F. Diele and I. Sgura, Isospectral flows and the inverse eigenvalue problem for Toeplitz matrices, J. Comput. Appl. Math., 110: 25-43 (1999).

[10] F. Diele and I. Sgura, The Cayley method and the inverse eigenvlue problem for Toeplitz matrices, BIT, 42: 285-299 (2002). 
[11] F. Diele, and T. Laudadio, and N. Mastronardi, On some inverse eigenvalue problems with Toeplitz-related structure, SIAM J. Matrix Anal. Appl., 26: 285-294 (2004).

[12] S. Friedland, J. Nocedal and M. L. Overton, The formulation and analysis of numerical methods for inverse eigenvalue problems, SIAM J. Numer. Anal., 24: 634-667 (1987).

[13] S. Friedland, Inverse eigenvalue problems for symmetric Toeplitz matrices, SIAM J. Matrix Anal. Appl., 13: 1142-1153 (1992).

[14] H. Gazzah, P. A. Regalia and J. Delmas, Asympototic eigenvalue distribution of block Toeplitz matrices and application to blind SIMO channel identification, IEEE Inform. Theory, 47: 1243-1251 (2001).

[15] G.H. Golub and C.F. Van Loan, Matrix Computations, Johns Hopkins University Press, Baltimore and London, 3rd edition, 1996.

[16] R. M. Gray, Toeplitz and circulant matrices, A review. Information theory laboratory, Stanford Univ. CA. [online]. Available: http://wwwisl.stanford.edu/gray/compression.html.

[17] U. Grenander and G. Szegö, Toeplitz forms and their applications, New York: Chelsea, 1984.

[18] R.D. Hill, and S.R. Waters, On $\kappa$-real and $\kappa$-Hermitian matrices, Linear Alg. Appl., 169: 17-29 (1992).

[19] H. J. Landau, The inverse eigenvalue problem for real symmetric Toeplitz matrices, J. American Math. Soc., 7: 401-405 (1994).

[20] D. P. Laurie, A numerical approach to the inverse Toeplitz eigenproblems, SIAM J. Sci. Stat. Comput., 9: 401-405 (1988).

[21] D. Laurie, Initial values for the inverse Toeplitz eigenvalue problem, SIAM J. Sci. Comput., 22: 2239-2255 (2000).

[22] Z.-Y. Liu, and H.-D. Cao, and H.-J. Chen, A note on computing matrixvector products with generalized centrosymmetric (centrohermitian) matrices, Applied. Math. Comput., 169: 1332-1345 (2005).

[23] D. Tao, and M. Yasuda, A spectral characterization of generalized real symmetric centrosymmetric and generalized real symmetric skew-centrosymmetric matrices, SIAM J. Matrix Anal. Appl., Vol. 23: 885-895 (2002).

[24] P. Tilli, On the asymptotic spectrum of Hermitian block Toeplitz matrices with Toeplitz blocks, Math. Computation, 66: 1147-1159 (1997).

[25] W.F. Trench, Numerical solution of the inverse eigenvalue problem for real symmetric Toeplitz matrices, SIAM J. Sci. Comput., 18: 1722-1736 (1997). 